ROCKY MOUNTAIN

JOURNAL OF MATHEMATICS

Volume 45, Number 6, 2015

\title{
VOLUME INDEX
}

Abu-Omar, Amer, Upper and lower bounds for the numerical radius with an application to involution operators, 1055.

Afkhami, M., Zero-divisor graphs of modules via module homomorphisms, 1.

Aiello, Domenico, Formal fibers with countably many maximal elements, 371.

Alexanderian, Alen, Expository Paper: A primer on homogenization of elliptic PDEs with stationary and ergodic random coefficient functions, 703.

Aquino, Cícero P., Characterizations of linear Weingarten spacelike hypersurfaces in Lorentz space forms, 13.

Arbieto, A., On various types of shadowing for geometric Lorenz flows, 1067.

Arnold, E., Nest graphs and minimal complete symmetry groups for magic Sudoku variants, 887.

Artés, J.C., From topological to geometric equivalence in the classification of singularities at infinity for quadratic vector fields, 29.

Ateş, Firat, see Karpuz, Eylem Güzel.

Awtrey, Chad, Degree 12 2-adic fields with automorphism group of order 4, 1755.

Ayad, Mohamed, On permutation binomials, 389.

Barkley, Brett, see Awtrey, Chad.

Bartłomiejczyk, Agnieszka, Existence and uniqueness of solutions for single-population McKendrick-von Foerster models with renewal, 401.

Belghaba, Kacem, see Ayad, Mohamed.

Bennis, Driss, On 2-SG-semisimple rings, 1093.

Bhandari, R., see Sharma, R.

Bilalov, B.T., Statistical convergence of functional sequences, 1413.

Binder, Thomas, Zeros of high derivatives of the Riemann zeta function, 903.

Bolaños, Yudy, Liouvillian first integrals for quadratic systems with an integrable saddle, 1765.

Boyle, Bernadette, The unimodality of pure O-sequences of type two in four variables, 1781.

Brown, Jim, Classifying extensions of the field of formal Laurent series over $\mathbb{F}_{p}, 115$.

Çevik, A. Sinan, see Karpuz, Eylem Güzel.

Chatzarakis, G.E., Asymptotic behavior in neutral difference equations with several retarded arguments, 131.

Chen, De-Han, New qualitative properties of solutions to nonlinear nonlocal Cauchy problems, 427.

Chen, Jianlong, see Di, Zhenxing.

Chen, Jinyuan, see Tang, Fengqin.

Cheng, Chunnuan, Normality concerning exceptional functions, 157.

Cheng, Sui Sun, see Huang, Shao Yuan.

Chmieliński, Jacek, A note on a characterization of metrics generated by norms, 1801.

$\mathrm{Chu}$, Jifeng, Nontrivial periodic solutions of second order singular damped dynamical systems, 457.

Csörgö, Piroska, All finite automorphic loops have the elementwise Lagrange property, 1101.

de Lima, Henrique F., see Aquino, Cícero P.

Deckelman, Steven, A multiplicative analogue of the Reynolds operator and construction of invariants, 1107. 
Decoste, Rachelle C., Totally geodesic subalgebras in 2-step nilpotent Lie algebras, 1425.

Delbourgo, Daniel, Transition formulae for ranks of abelian varieties, 1807.

Demeter, Ciprian, A guide to Carleson's theorem, 169.

Demeyer, Lisa, see Decoste, Rachelle C.

Deng, Xun-Huan, Nonoscillatory solutions to forced higher-order nonlinear neutral dynamic equations on time scales, 475.

Di, Zhenxing, Stability of Gorenstein flat categories with respect to a semidualizing module, 1839.

Ding, Ming-ling, see Xiao, Xiang-chun.

Estaji, E., see Afkhami, M.

Felipe, Raúl, see Trejo, Imelda.

Field, R., see Arnold, E.

Gallaspy, Michael, The Goeritz matrix and signature of a two bridge knot, 1119.

Gil', Michael, Norm estimates for functions of two non-commuting operators, 927.

Golbaharan, A., Essential spectral radius of quasicompact endomorphisms of Lipschitz algebras, 1149.

Graetz, Jennifer, see Deckelman, Steven.

Grimme, Felix, All exponential moments of the Dyck paths maxima (scaled by root of path length) are uniformly bounded: An elementary proof, 1861.

Hasmani, Alfeen, see Brown, Jim.

Hernández-Gutiérrez, Rodrigo, Rigidity of hyperspaces, 213.

Hiltner, Lindsey, see Brown, Jim.

Hirata-Kohno, Noriko, On the Diophantine equation $F_{n}^{x}+F_{n+1}^{x}=F_{m}^{y}, 509$.

Hitchman, Michael P., The topology of tile invariants, 539.

Hou, Jinchuan, see Qi, Xiaofei.

$\mathrm{Hu}$, Kui, see Bennis, Driss.

Huang, Shao Yuan, Existence of eventually positive solutions of higher order impulsive delay differential equations, 237.

Humphries, Stephen P., A class of groups determined by their 3-S-rings, 565.

Illanes, Alejandro, see Hernández-Gutiérrez, Rodrigo.

Izuchi, Kei Ji, Topological properties of path connected components in spaces of weighted composition operators into $L^{\infty}, 941$.

Izuchi, Yuko, see Izuchi, Kei Ji.

Jabuka, Stanislav, see Gallaspy, Michael.

Jacobson, Jr., M.J., see Rozenhart, P.

Karpuz, Eylem Güzel, Gröbner-Shirshov bases of some Weyl groups, 1165.

Karr, Ryan, Picard groups and torsion-free cancellation for orders in $\mathbb{Z} \times \mathbb{Z} \times \mathbb{Z}, 1873$.

Katsevich, Anya, On De Graaf spaces of pseudoquotients, 1445.

Kawamura, Kazuhiro, An abelian group associated with topological dynamics, 1457.

Khan, Viqar Azam, see Uddin, Siraj.

Khashyarmanesh, K., see Afkhami, M.

Khorsandi, M.R., see Afkhami, M.

Kiani, Dariush, On the addition of units and non-units in finite commutative rings, 1887.

Kihel, Omar, see Ayad, Mohamed.

Kikuta, Toshiyuki, Note on Igusa's cusp form of weight 35, 963.

Kittaneh, Fuad, see Abu-Omar, Amer.

Kodama, Hirotaka, see Kikuta, Toshiyuki.

Koşan, M. Tamer, see Quynh, Truong Cong.

Kosta, Neža Mramor, see Zlobec, Borut Jurčıč. 
Kraft, Angela, see Brown, Jim.

Krantz, Steven G., The boundary behavior of holomorphic functions, 1471.

Lanski, Charles, Finite index conditions in rings, 1177.

Lecacheux, Odile, Weierstrass equations for all elliptic fibrations on the modular K3 surface associated to $\Gamma_{1}(7), 1481$.

Lee, Keun Young, New criteria for the weak Radon-Nikodým property related to set-valued operators, 1511.

Lei, Antonio, see Delbourgo, Daniel.

Leszczyński, Henryk, see Bartłomiejczyk, Agnieszka.

Lewis, Mark L., Erratum on "Classifying Camina groups: A theorem of Dark and Scoppola, ” 273.

Li, Shengjun, see Chu, Jifeng.

Li, Zehui, see Tang, Fengqin.

Lian, Wei-Cheng, see Wong, Fu-Hsiang.

Lin, Ren-Ci, see Wong, Fu-Hsiang.

Lin, Weichuan, see Pan, Biao.

Liu, Zhongkui, see Di, Zhenxing.

Liu, Zhongkui, see Zhang, Chunxia.

Llibre, J., see Artés, J.C.

Llibre, Jaume, see Bolaños, Yudy.

Loepp, S., see Aiello, Domenico.

Lorch, J., see Arnold, E.

Luca, Florian, see Hirata-Kohno, Noriko.

Lucas, S., see Arnold, E.

Mahanti, Mina Ketan, On expected number of level crossings of a random hyperbolic polynomial, 1197.

Mahyar, H., see Golbaharan, A.

Malmskog, Beth, The local and global zeta functions of Gauss's curve, 275.

Mao, Lixin, Precovers and preenvelopes under change of rings, 1527.

Marsh, M.M., Internal inverse limits and retractions, 1209.

Martínez-de-la-Vega, Verónica, see Hernández-Gutiérrez, Rodrigo.

Martínez-Martínez, Javier, The SU(2)-character varieties of torus knots, 583.

Martini, Horst, Orthogonalities, transitivity of norms and characterizations of Hilbert spaces, 287.

Matkowski, Janusz, Some characterizations of the Euler gamma function, 1225.

Matuła, Przemysław, On Etemadi's subsequences and the strong law of large numbers for random fields, 1555.

Melman, A., Geometric aspects of Pellet's and related theorems, 603.

Mikić, Miljen, On the Mordell-Weil group of elliptic curves induced by families of Diophantine triples, 1565.

Mikusiński, Piotr, see Katsevich, Anya.

Miles, Nicole E., see Awtrey, Chad.

Miliaras, G.N., see Chatzarakis, G.E.

Miyasita, Tosiya, A dynamical system for a nonlocal parabolic equation with exponential nonlinearity, 1897.

Miyazaki, Takafumi, Upper bounds for solutions of an exponential Diophantine equation, 303.

Mollahajiaghaei, Mohsen, see Kiani, Dariush.

Moody, Dustin, Character sums determined by low degree isogenies of elliptic curves, 623 . 
Mooney, Christopher Park, Generalized U-factorization in commutative rings with zero-divisors, 637.

Muñoz, Vicente, see Martínez-Martínez, Javier.

Muskat, Jeremy, see Malmskog, Beth.

Nagaoka, Shoyu, see Kikuta, Toshiyuki.

Nazarova, T.Y., see Bilalov, B.T.

Niemiec, Piotr, Elementary approach to homogeneous $C^{*}$-algebras, 1591.

Ohno, Shûichi, see Izuchi, Kei Ji.

Overmoyer, Kate, Non-Synthetic diagonal operators on the space of functions analytic on the unit disk, 1233.

Ozel, Cenap, see Uddin, Siraj.

Pan, Biao, Angular value distribution concerning shared values, 1919.

Pauli, Sebastian, see Binder, Thomas.

Pilipović, Stevan, On a solution of the Cauchy problem in the weighted spaces of Beurling ultradistributions, 1937.

Poulios, Costas, The fixed point property and the Opial condition on treelike Banach spaces, 1245.

Prajs, J.R., see Marsh, M.M.

Prangoski, Bojan, see Pilipović, Stevan.

Qi, Xiaofei, Full-derivable points of $\mathcal{J}$-subspace lattice algebras, 345.

Qi, Yuan, see Wen, Zijuan.

Quynh, Truong Cong, On the substructures $\Delta$ and $\nabla, 661$.

Rao, Arni S.R. Srinivasa, Incubation periods under various anti-retroviral therapies in homogeneous mixing and age-structured dynamical models: A theoretical approach, 973.

Rasmussen, Christopher, see Moody, Dustin.

Reis, J.E., see Arbieto, A.

Ribeiro, R., see Arbieto, A.

Rode, Emma L., see Humphries, Stephen P.

Rozenhart, P., Computing quadratic function fields with high 3-rank via cubic field tabulation, 1985.

Sadeghi, Ghadir, Inequalities for sums of independent random variables in Lorentz spaces, 1631.

Sahoo, Loknath, see Mahanti, Mina Ketan.

Saidak, Filip, see Binder, Thomas.

Scheidler, R., see Rozenhart, P.

Schlomiuk, D., see Artés, J.C.

Scofield, Daniel, see Brown, Jim.

Seubert, Steven M., see Overmoyer, Kate.

Seweryn, Michał, see Matuła, Przemysław.

Sharma, R., Skewness, kurtosis and Newton's inequality, 1639.

Shill, Christopher, see Awtrey, Chad.

Shu, Zhi-biao, see Xiao, Xiang-chun.

Smith-Tone, Daniel, A measure of dependence for cryptographic primitives relative to ideal functions, 1283.

Stankov, Dimcho K., Pseudo-hyperbolic distance and Gleason parts of the algebra of bounded hyper-analytic functions on the big disk, 1033.

Strosnider, Erin, see Awtrey, Chad.

Sun, Jingxian, see Xing, Hui.

Sun, Jiong, see Zettl, Anton.

Taalman, L., see Arnold, E. 
Tang, Fengqin, Precise large deviations of aggregate loss process in a risk model based on the policy entrance process, 2023.

Tone, Cristina, see Smith-Tone, Daniel.

Tong-Viet, Hung P., Prime divisors of irreducible character degrees, 1645.

Trejo, Imelda, The spectrum in $\mathbf{R}$ and $\mathbf{R}^{2}$ of nonlinear elliptic equations with positive parameters, 1311.

Tyler, Russell, see Deckelman, Steven.

Uddin, Siraj, Classification of totally umbilical $\xi^{\perp}$ CR-submanifolds of cosymplectic manifolds, 361 .

Valls, Claudia, see Bolaños, Yudy.

Velásquez, Marco Antonio L., see Aquino, Cícero P.

Velinov, Daniel, see Pilipović, Stevan.

$\mathrm{Vu}$, Philip, see Aiello, Domenico.

Vulpe, N., see Artés, J.C.

Wang, Fanggui, see Bennis, Driss.

Wang, Limin, see Zhang, Chunxia.

Wang, Qi-Ru, see Deng, Xun-Huan.

Wang, Rong-Nian, see Chen, De-Han.

Wash, Kirsti, see Brown, Jim.

Weiss, Ittay, Survey Article: The real numbers-A survey of constructions, 737.

Wen, Zijuan, Non-constant positive steady states for a strongly coupled nonlinear reaction-diffusion system arising in population dynamics, 1333.

Wong, Fu-Hsiang, Existence of solutions for the generalized p-Laplacian equation, 1047.

$\mathrm{Wu}$, Senlin, see Martini, Horst.

$\mathrm{Xu}$, Yan, see Cheng, Chunnuan.

Xiao, Xiang-chun, G-frames with bounded linear operators, 675.

Xiao, Xiaoyong, Oscillations and moduli of continuity of kernel density estimators under dependence, 1659.

Xiao, Zhankui, Jordan derivations of incidence algebras, 1357.

Xing, Hui, Computation of fixed point index and its applications, 1369.

Yang, Xiaoyan, Gorenstein categories $\mathcal{G}(\mathscr{X}, \mathscr{Y}, \mathscr{Z})$ and dimensions, 2043.

Yin, Hongwei, see Xiao, Xiaoyong.

Yuan, Haiping, New families of weighted sum formulas for multiple zeta values, 2065.

Zaare-Nahandi, Rashid, Pure simplicial complexes and well-covered graphs, 695.

Zettl, Anton, Survey Article: Self-adjoint ordinary differential operators and their spectrum, 763.

Zhang, Chunxia, Ding projective modules with respect to a semidualizing bimodule, 1389.

Zhang, Mingji, Asymptotic expansions and numerical simulations of I-V relations via a steady state Poisson-Nernst-Planck system, 1681.

Zhao, Jianqiang, see Yuan, Haiping.

Zhu, Hailong, see Chu, Jifeng.

Zhu, Yu-can, see Xiao, Xiang-chun.

Zlobec, Borut Jurčıč, Geometric constructions on cycles in $\mathbb{R}^{n}, 1709$.

Zwierkowski, Piotr, see Bartłomiejczyk, Agnieszka. 